\title{
Traduire
}

Revue française de la traduction

$232 \mid 2015$

Intraduisible? Vous voulez rire !

\section{Doublage et duplicité : traduire l'humour verbal au cinéma}

\section{Frédérique Brisset}

\section{(2) OpenEdition}

Journals

\section{Édition électronique}

URL : http://journals.openedition.org/traduire/688

DOI : $10.4000 /$ traduire.688

ISSN : 2272-9992

\section{Éditeur}

Société française des traducteurs

\section{Édition imprimée}

Date de publication : 15 juin 2015

Pagination : 5-21

ISSN : 0395-773X

\section{Référence électronique}

Frédérique Brisset, «Doublage et duplicité : traduire l'humour verbal au cinéma », Traduire [En ligne], 232 | 2015, mis en ligne le 15 juin 2017, consulté le 03 mai 2019. URL : http://journals.openedition.org/ traduire/688 ; DOI : 10.4000/traduire.688 


\section{Doublage et duplicité : traduire I'humour verbal au cinéma}

\section{Frédérique Brisset}

"Ta blague, c'est du chinois " : ce titre repris par France Culture(1) synthétise la problématique sous-jacente à la réception de l'humour. II faut en effet souvent décoder ce dernier comme une langue étrangère, du fait de sa duplicité. La duplicité, selon le Trésor de la Langue Française Informatisé, caractérise " 1. ce qui est double, 2. le fait d'avoir un comportement double, variant selon les circonstances ". Au cinéma, le doublage de l'humour, et des jeux de mots en particulier, se heurte à un summum de duplicité, le décodage intralinguistique du fait humoristique, lié à la seconde acception du terme, se combinant au décodage interlinguistique, qui résulte de la première. (La frontière entre humour et comique étant relativement imprécise, ces termes sont utilisés ici comme quasi synonymes, car ils ont un intérêt sur un plan pragmatique, en fonction des effets recherchés.)

Woody Allen, par exemple, affectionne depuis longtemps une forme d'humour verbal héritée de ses débuts de carrière en tant que stand-up comedian(2). Ses dialogues, reconnus pour leur écriture très " orale " et le recours à la langue comme instrument ludique vecteur de dérision, ont construit un personnage dont l'obsessive verbosité provoque immanquablement le rire du spectateur. Ce dernier attend donc des répliques à visée comique et sa coopération, déterminante pour que se produise l'effet attendu à la réception, est anticipée et sollicitée par le cinéaste-scénariste. Car c'est dans l'interaction que l'humour est appelé à fonctionner, interaction dans les échanges entre protagonistes à l'écran, mais surtout avec le public co-constructeur du sens. Quand le film est traduit pour l'étranger, notamment pour le doublage, ce destinataire ultime n'est plus le même, mais les répliques doivent atteindre la même efficacité pour déclencher le rire voulu par leur auteur.

(1) Le rire dans tous ses éclats, 2/4, "Ta blague, c'est du chinois ", CulturesMonde, France Culture, 24 avril 2012.

(2) " La stand-up comedy allénienne est, bel et bien, à l'aune de l'ensemble de l'œuvre. [...] Meneur de jeu et public sont réunis sous un même toit pour participer à une même cérémonie au cours de laquelle on rit, tous ensemble ", Dreyfus Jean-François, 1999, "L'humour dans les écrits de Woody Allen ", Journal of the Short Story in English, 32, p. 3. 


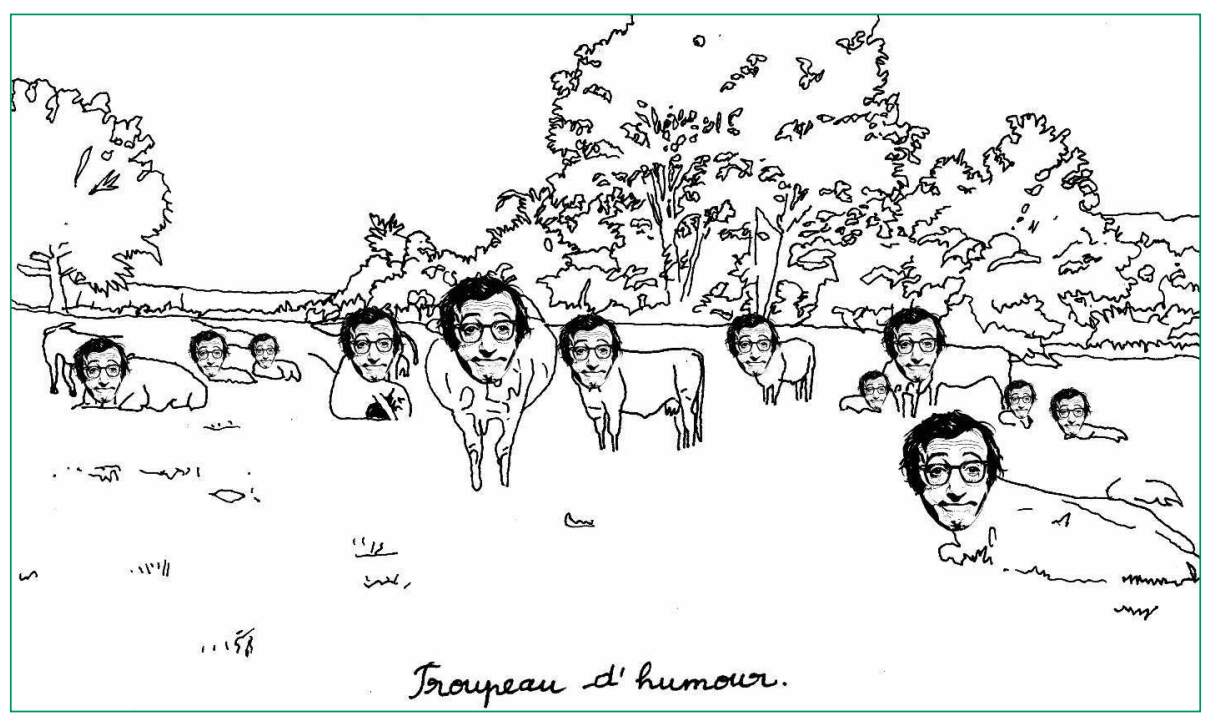

Illustration de Nicolas Lefebvre - www.nikopoisson.tumblr.com - nikopoisson@gmail.com

Dans ce processus, la catégorie des jeux de mots, notamment, pose un problème traductif majeur, du fait de l'essence du procédé, le recours à l'ambiguité par la mise à mal de l'univocité du signifiant : " II apparaît comme le résultat d'une série de rapports ponctuels entre signifiants et signifiés, dits et non-dits " (Raynaud, 1977, 24). Le médium filmique, de par sa composante orale, se prête d'autant mieux à cet exercice que le sens qu'il convoie est, au-delà de l'image, basé aussi sur des phénomènes phonétiques.

Conjuguée aux contraintes linguistiques du transfert de l'anglais au français et aux problèmes techniques inhérents au mode du doublage, la duplicité du jeu de mots présente des difficultés majeures pour les professionnels de la traduction audiovisuelle. Leur travail d'adaptation fournit pourtant des solutions souvent fort habiles pour son transfert interlinguistique.

Quelques exemples tirés de quatre fims d'Allen couvrant 25 années de sa filmographie, Everything You Always Wanted to Know About Sex (But Were Afraid to Ask) (1972), Annie Hall (1977), Radio Days (1987) et Deconstructing Harry (1997), sont ici comparés avec leurs versions françaises doublées et/ou publiées(3), pour comprendre les stratégies des traducteurs-adaptateurs face à ces manipulations ludiques de la langue, qui construisent un tissu humoristique très dense.

(3) cf. bibliographie, abréviations utilisées : VO : version originale, VD : version doublée, DV : D. Valion, GD : G. Dutter, JC : J. Cohen, ML : M. Lebrun). V0 référencée par le minutage, extraits publiés, par leur pagination. 


\section{Dupliquer la duplicité}

Le jeu de mots implique une approche consciente par l'énonciateur de la structure de la langue qu'il utilise, de ses bases phonologiques et des phénomènes de synonymie : "Rien ne sépare mieux le mot d'esprit de toutes les autres formations psychiques que cette double face, ce double langage qui sont les siens [...] " (Freud, 1988, 310). Ce fondement purement verbal fait que la catégorie des jeux de mots pose un problème traductif considéré par beaucoup de commentateurs comme insoluble(4), au point que Le Robert a choisi d'illustrer son entrée " traduisible " par la phrase : "Ce jeu de mots n'est guère traduisible(5)".

De nombreux spécialistes se sont confrontés à cette problématique. Durastanti $(2002,112)$ estime que " la différence [d'une langue à l'autre] rend la traduction difficile, et les jeux de mots souvent intraduisibles ", tandis que Dupriez $(1984,270)$ affirme : "Le jeu de mots succombe à la traduction ", tout en reconnaissant : "Cependant, certains traducteurs, ceux d'Ulysse notamment, ont fait des prodiges. "Eco $(2006,111)$ considère : " II y a des pertes dites absolues. Ce sont les cas où il est impossible de traduire [...]. Un exemple de perte absolue est le jeu de mots ". Desmond $(2005,87)$ insiste de même sur la résistance usuelle du calembour à la traduction, justifiant en conséquence son occultation. Bergson (2002, 79), avant eux, considérait que l'humour résultant de choix syntaxiques ou lexicaux est en général intraduisible. Quant à Berman (1984, 190), s'intéressant au lien entre poésie et traduction, il disait " douter qu'il soit plus facile de traduire des jeux de mots que des "jeux de sonorités" ".

Ces divers arguments reposent tous sur la structuration même des jeux de mots ; mais il en est d'autres liés à leur transfert de l'anglais au français, du fait des spécificités de chaque langue : Eco $(2006,360)$ distingue ainsi " une langue docile au pun, au néologisme et à l'emboîtement des mots telle que l'anglais (favorisé par l'abondance de termes monosyllabiques) ". Suhamy $(1994,106)$ insiste en outre sur le statut spécifique du jeu de mots en anglais, comparé au français :

II ne sert pas seulement à déclencher le rire par un effet de surprise et d'incongruité. [...] II implique souvent une prise de distance à l'égard des règles contraignantes du langage, une subversion du code, une exploration aventureuse des ressources cachées de la langue.

Lorsque l'on ajoute à ces différents constats les exigences de synchronisation et de cohérence image-son qui contraignent le doublage, l'enjeu est donc multiple :

Woody Allen est un cauchemar pour notre métier. Les répliques, bourrées de jeux de mots intraduisibles, nous obligent à en inventer d'autres en français. Comme nous le disons dans notre jargon, il faudra pour autant que nos mots "rentrent en bouche " de l'acteur, c'est-à-dire s'inscrivent dans le temps de parole, comme dans les mouvements de lèvres du comédien (Corneloup, 2011, 34-35).

(4) À rapprocher, sur ce plan, de la poésie, fondée sur une combinaison du sens et du son lui accordant " le glorieux privilège de l'intraduisibilité ", comme l'écrit Gérard Genette dans Palimpsestes (Paris, Éditions du Seuil, 1982, p. 239).

(5) Micro-Robert, dictionnaire du français primordial, Paris, Le Robert, 1981. 
Lors d'un entretien personnel en février 2011, Jacqueline Cohen, adaptatrice d'Allen de 1989 à 2012, exprimait d'ailleurs son soulagement de n'avoir pas eu à traiter ses films de début de carrière, traduits par Georges Dutter dès 1977, car leurs dialogues regorgeaient de jeux de mots. II n'est donc pas étonnant qu'apparaisse chez Benayoun $(1985,15)$ le fameux " privilège " d'intraduisibilité : "Notons que Woody est le seul comique de l'histoire du cinématographe dont la parole exige la v.o. dans toutes ses nuances [...] : sa langue concise, télescopée, faite d'aphorismes demande en français deux fois plus de mots qu'en anglais".

Si certains jeux de mots obligent l'adaptateur à se cantonner effectivement à une stratégie de compensation, ils n'offrent pourtant pas tous la même difficulté traductive ; cela tient en partie à leur construction linguistique, mais aussi à leur contexte d'apparition et à leur fonction dans l'œuvre considérée. Allen alerte d'ailleurs ses traducteurs sur ces unités problématiques dans la continuité dialoguée qui leur est fournie : «It makes transparent any play on words or possible ambiguity, explains the meaning of colloquial or dialectal words and exemplifies the meaning of all proper names " (Díaz Cintas, 1998, 55-56). Ces aides leur permettent d'effectuer les repérages indispensables afin de trouver des solutions adéquates pour le doublage de ces jeux de mots et réfuter de facto le postulat d'intraduisibilité.

On a donc plutôt affaire à des cas limites, qui infirment le jugement assez péremptoire et mal fondé d'un biographe d'Allen sur la VD française d'Annie Hall : " Half the jokes weren't translatable, so the writer of the French version didn't even try " (Baxter, 1999, 2). Le transfert en français de ce comique verbal peut s'avérer ardu, mais il est rare que les traducteurs s'en affranchissent alors que ces idiosyncrasies sont l'une des clés du succès d'Allen. Dutter (1981, 446-447) résume l'alternative en une métaphore chimique :

[...] il n'y a pas d'échappatoire possible pour le traducteur : ou il donne le sens littéral, et là, la connivence tombe comme un précipité au fond du tube. Et c'est l'humour "qui y reste ". Ou alors, il agite les mots, et la lecture deviendra approximative. Encore qu'au goût, la saveur reste marquée... et même bonne.

Pour l'analyse de la traduction des jeux de mots, nous utilisons la taxonomie de Jacqueline Henry $(2003,176)$, qui partage en quatre classes les processus traductifs (isomorphe, homomorphe, hétéromorphe ou libre, fondés " sur le maintien ou non du même procédé que dans l'original "), dont trois sont décrits au fil des exemples commentés ci-après. Ces derniers sont rassemblés selon les procédés linguistiques auxquels ils ont recours(6), même si la typologie des jeux de mots n'offre pas toujours de distinction tranchée entre ces diverses catégories(7).

(6) cf. Dirk Delabastita, 1996, Wordplay and Translation, The Translator, vol. 2, 2, p. 128, sur la gradation des procédés : homonymie, homophonie, homographie, paronymie,

(7) cf. Raynaud $(1977,28)$ : "Assurément, rares sont les jeux linguistiques qui peuvent aisément se laisser ranger à l'intérieur de catégories "pures". " 


\section{Duplicité polysémique}

La première classe de jeux de mots présentée dans cette partie est basée sur la double acception d'items lexicaux, phénomène ludique exploité par Allen comme par maints auteurs anglophones(8).

Ils servent à la caractérisation de personnages malhabiles qui se risquent à des plaisanteries plus ou moins heureuses pour se donner une contenance, tels le bouffon du premier sketch de All You Always Wanted to Know About Sex (1972), - Suhamy $(1994,106)$ relève d'ailleurs "la bouffonnerie intrinsèque du jeu de mots "-, Joey, l'ami de la famille de Annie Hall (1977) ou le maître de cérémonie de Radio Days (1987) rencontrés dans cet article, mais aussi à des jeux d'interprétation plus subtils, où le locuteur utilise la pirouette verbale afin d'éluder une question de fond dans l'échange conversationnel. Dans les deux cas, sur le plan pragmatique, le jeu de mots marque une tentative du protagoniste de garder la face et le dialogue dramatique imite un rituel de la conversation quotidienne. Mais au niveau auctorial de l'énonciation, il signale fréquemment une volonté artistique de provocation et de transgression des codes sociaux.

\subsection{Hongrois toujours avoir compris...}

Pour le bouffon d'une cour royale, le jeu de mots relève quasiment de l'obligation professionnelle. Dans la continuité de l'alter ego de gaffeur récidiviste introduit dans ses premiers films, Allen met en scène dès l'ouverture de Tout ce que vous avez toujours voulu savoir... un fou du roi on ne peut plus maladroit.

V0, 4'40, Fool: That, that plague is really something, isn't it? Doesn't everything look black? Coz it's a bla..., a black, a black plague.

Dans ce sketch parodique, la plaisanterie, laborieuse, provoque la consternation de la cour puis la colère royale, d'autant qu'elle renvoie à un sujet tragique, l'épidémie qui a marqué l'histoire du Vieux Continent. Lebrun, traducteur de la version française publiée, opte pour la traduction littérale de plague:

ML, 16, Le fou : ... euh... La peste, c'est vraiment un sale truc, pas vrai ? C'est noir, ça rend boudeur, d'ailleurs ça vient de l'étranger... d'où l'expression «boude à peste ". (II rit, il est le seul.)

La construction de la locution "boude à peste ", collocation improbable évoquant par homophonie la cité hongroise, combine deux lexèmes introduits dans le co-texte anaphorique immédiat, sur le même principe que la V0, mais elle s'avère plus sophistiquée, puisque l'original

(8) "La polysémie et l'homonymie sont des sources inépuisables de jeux de mots qui alimentent les calembours et malentendus de la vie quotidienne et que la littérature a largement exploités, de Shakespeare à Lewis Carroll ", Michel Paillard, 2000, Lexicologie contrastive anglais-français, Gap, Ophrys, p. 128. 
se contentait d'associer un nom et un adjectif ; c'est néanmoins une traduction homomorphe, " procédé de traduction par lequel l'original est rendu par un jeu de mots du même type [...] mais fondé sur des termes qui ne correspondent pas directement à ceux du jeu verbal de départ " (Henry, 2003, 290). Le bouffon se sent obligé d'expliquer l'élaboration du jeu de mots, or un jeu de mots qui nécessite un tel décodage signe l'échec de son énonciateur, puisqu'il met à mal sa duplicité et échoue à établir la connivence indispensable à sa réception. L'effet produit est conforme à celui du script initial, comme le confirme la didascalie qui suit : " II rit, il est le seul ". La VD, dont l'adaptateur n'a pu être identifié, a recours à une autre équivalence, basée sur un mot-valise :

VD : [...] La peste, la peste c'est vraiment un truc terrible. Paraît que ça vient d'Asie... eh, c'est c'qu'on appelle Bouddha... Bouddha-peste.

Cette traduction fait à nouveau appel à la capitale hongroise, mais le jeu de mots-valise convoque maintenant une divinité indienne et le qualificatif " noir " a été supprimé. Les deux versions françaises aboutissent à la même efficacité que le dialogue originel en ce qui concerne la caractérisation du bouffon et son effet sur l'auditoire diégétique royal, mais toute correspondance lexicale avec la version américaine a disparu. Elles répondent cependant aux critères du jeu de mots selon Bergson $(2002,144)$ : " les relations établies entre les idées deviennent plus superficielles : peu à peu nous arrivons à ne plus tenir compte du sens des mots entendus, mais seulement du son."

\subsection{Quand la perche plie}

Dans certaines occasions, le traducteur offre des solutions beaucoup plus sophistiquées, comme dans cet extrait de Radio Days où Bea, candidate d'un jeu radiophonique où elle devait reconnaître des poissons, est félicitée par l'animateur. Version contemporaine du bouffon médiéval du précédent exemple, il use de la plaisanterie comme d'un rituel professionnel :

V0, 66'50, MC: And finally, how about this one?

Bea: That, that's a fl... floun... flounder. No, no, no. That's a fluke.

MC: You're sure?

Bea: That's a fluke.

MC: Well, this is no fluke, you've won fifty silver dollars!

Le sens premier de fluke est celui de " plie " ou " carrelet ", dans le technolecte ichtyologique. Pour apprécier la plaisanterie, il faut décoder le sens figuré, argotique, employé par l'animateur en conclusion : a failure ou a fortuitous accident; a freak success that could not be repeated (Pocket Dictionary of American Slang). II n'y a en effet, nul hasard dans le fait que Bea a pu identifier la série de poissons en plastique exposés sur scène : son beau-frère rapporte tous les jours à la maison les prises de ses amis pêcheurs. 
Le traducteur doit donc retrouver en français une expression adéquate, organisée sur la confusion entre les deux isotopies que sont les sens propre et figuré du terme, et issue du même champ lexical ; ces conditions sont nécessaires pour respecter le co-texte, le contexte et la visée pragmatique de la séquence, qui met en scène un maître de cérémonie adepte de jeux de mots passablement éculés.

VD, Présentateur : Et pour finir, ça s'appelle comment?

Bea: Ce, ce, c'est un fle, flé, flé... tan. Non, non. C'est une grosse perche.

Présentateur: Vous êtes sûre?

Bea : C'est une perche.

Présentateur: Et je ne lui ai pas tendu la perche ! C'est gagné, vous avez gagné cinquante dollars !

Dutter réussit à satisfaire toutes les conditions d'équivalence, en créant un jeu de mots élaboré sur le même principe que l'original, I'homonymie, dans le même registre lexical, avec le recours à la locution figée " tendre la perche ". II ne peut toutefois parvenir à une traduction isomorphe qui maintiendrait le sémantisme : il modifie donc l'espèce du poisson en transformant la plie en perche, poisson d'eau douce qui permet un calembour(9) phonique assez facile, respectant la caractérisation de l'animateur et la visée pragmatique du dialogue. Comme celui de la V0, le spectateur de la VD peut alors sourire, voire rire, en entendant la plaisanterie, et partager la réaction de l'auditoire interne au film, puisque l'émission radiophonique est enregistrée en public.

La démarche relève de la traduction homomorphe, mais illustre les limites de la transparence du doublage : ce qui est transparent "ne fait pas écran à la vision " (Trésor de la Langue Française Informatisé), et, l'animal étant visible à l'écran, le doubleur a veillé à ajouter le qualificatif " grosse " au nom "perche " dans la première réplique de Bea. La comédienne est filmée sur scène avec l'animateur et l'orchestre en plan américain, l'ajout de cet adjectif dans sa réponse pose peu de problèmes pour la synchronisation labiale. Cette manipulation linguistique permet de justifier la différence de morphologie entre l'espèce montrée presqu'à contre-jour à l'écran, un grand poisson plat, et celle reconnue oralement par le personnage, mais aussi son hésitation avec le "flétan ". Le doubleur, grâce à ce compromis, aboutit à une cohérence satisfaisante entre la bande-son et l'image, qui ne remet pas en cause la vraisemblance de la diégèse, même si le sémantisme du référent a été sacrifié dans l'opération.

Dutter $(1981,446)$ résume parfaitement le choix traductif qui s'offre dans ce type de situation : "Alors, empirique, on se dit qu'on va adapter : I'humour, c'est fait... pour rire. Donc, on va faire appel à des produits de remplacement. " La cohérence est ensuite maintenue dans cet épisode, puisqu'en fin de journée, Bea se remémore ainsi l'épisode :

V0, 67’30, Bea: God, I almost forgot what a fluke looked like.

VD, Bea : Oui, dire que j'ai presque eu un trou pour cette perche !

(9) "Énoncé contenant un élément à plurivalence sémique ou phonique " (Henry, 2003, 288). 


\subsection{Blanc cassé}

Le jeu sur les anthroponymes est récurrent dans la filmographie d'Allen. Freud $(1988,64)$ le soulignait déjà : " D'une manière générale, les noms propres se prêtent facilement au traitement que la technique du mot d'esprit leur fait subir. " Généralement implicite, car fondé sur le double sens et la connotation, il peut pourtant donner lieu à des plaisanteries ouvertement revendiquées. Le titre d'une émission de ragots sur les célébrités en offre un bel exemple, toujours dans Radio Days.

V0, 61', Speaker: And now, the makers of Lady Lydia facial cream bring you Sally White and Her Gay White Way.

L'intitulé joue sur la polysémie de White, tout à la fois patronyme de la chroniqueuse radiophonique et adjectif qualificatif homonyme. II renvoie implicitement à la qualité du produit de la marque sponsor de l'émission, une crème faciale censée purifier le teint, et à un sens second, argotique, glosé par une longue liste de synonymes dans le Pocket Dictionary of American Slang : ethical, honest, fair, faithful, dependable, decent, friendly, regular.

L'ironie résulte du fait que l'angle choisi par Sally n'est nullement fidèle à cette éthique éditoriale, puisqu'elle jase sur la vie privée de vedettes du spectacle (l'émission s'ouvre par une indiscrétion sur Clark Gable escortant une pimpante brune au club El Morocco). Le report du titre américain du programme ne fonctionnerait pas en VD, car il ne permettrait pas au spectateur francophone de comprendre l'ironie sous-jacente, pas plus qu'une traduction littérale. Le doubleur conserve donc un jeu sur l'anthroponyme, mais exploite le prénom de l'animatrice, tout en explicitant le contenu de sa chronique:

VD, Présentateur: Maintenant les produits de beauté de Lady Lydia proposent Sally White et son "Qui n'ai-je pas sali ?".

Ce jeu de mots repose sur l'homophonie entre Sally et sali ; pourtant le titre final semble improbable : il est peu envisageable qu'une émission affiche ostensiblement cet objectif de dénigrement systématique. On a ici une équivalence d'effet quant au jeu de mots, mais l'efficacité dramatique est moindre, car la traduction retenue remet en cause la vraisemblance du scénario. Alors que le sous-titrage se limite au syntagme hyperonyme "chronique mondaine ", le doublage privilégie ici la composante phonétique en occultant le titre initial de l'émission. Dans ce compromis traductif, le doubleur a accordé la priorité au trait d'humour, avec une traduction homomorphe qui moque ce genre d'émission cancanière.

\subsection{Pince-sans-rire}

La traduction homomorphe n'est pas toujours facile, et le traducteur doit parfois se contenter d'un jeu de mots relevant d'un procédé différent de l'original, comme dans cette scène de Annie Hall qui montre Alvy et Annie occupés à rattraper des homards échappés dans leur 
cuisine. Lors de cet exercice filmé en plan moyen avec de nombreux mouvements panoramiques, elle se révèle nettement plus efficace que lui, caractérisé par sa phobie de la nature en général, et de la faune en particulier.

V0, 18', 46, Alvy: (Laughing) Talk to him. You speak shellfish!

La plaisanterie relève ici du nonsense, puisqu'il n'existe pour dialoguer entre humains et homards nulle langue crustacée, traduction littérale de speak shellfish, - c'est pourtant le choix de la VOST qui affiche "Tu parles le crustacé " -, même si shellfish partage le suffixe -ish de noms de langue tels que English, Irish, Finnish, Turkish, Polish, etc. Devant l'impossibilité de produire un syntagme français sur le même fondement, les traducteurs du scénario, Valion tout comme Dutter, renoncent à ce registre humoristique pour un autre jeu de mots :

DV, 15, Alvy: (mi-effrayé, mi-amusé) Parle-lui ; toi qui en pinces pour lui !

GD, 47, Alvy: (il se met à rire aussi) Parle-lui, toi qui en pinces pour lui !

Le calembour in absentia joue sur la proximité entre les pinces, élément anatomique du homard, et l'expression argotique " en pincer pour ", synonyme de "avoir un penchant pour ". La dimension comique est renforcée en VF avec cette traduction hétéromorphe, " traduction d'un jeu de mots par un jeu de mots utilisant un autre procédé que l'original " (Henry, 2003, 290). La VD étoffe encore la proposition dans cet épisode, le plan de profil cadré à la taille imposant une synchronisation somme toute relative :

VD, Alvy: Parle-lui, toi qui en pinces pour ceux qui savent nager !

La répartie d'Alvy, plus longue qu'en V0, passe de sept à onze syllabes dans cette séquence pourtant très rythmée, sans que l'étoffement, allusion à la recette du homard à la nage, n'apporte d'élément pertinent ni pour l'équivalence d'effet, ni sur la caractérisation des personnages, ni même pour la progression de l'intrigue. II y a ici une surtraduction du jeu de mots transformant l'acteur Allen en un clown qui relève plutôt de sa filmographie antérieure.

Ces différents exemples sont représentatifs de la capacité d'autodérision d'Allen ; mais en parodiant un humour qui paraît en surface assez inefficace car basé sur des jeux de mots laborieux, il joue aussi sur le second degré. Cette fonction est généralement bien appréhendée par les traducteurs : plus la ficelle est grosse, plus il leur est aisé d'identifier et de transposer ce comique verbal.

\section{Duplicité paronymique}

Les jeux de mots construits sur de quasi-homophonies sont un autre procédé ludique récurrent chez Allen, car la langue anglaise se prête particulièrement à la formation de doublets lexicaux sur des similitudes phoniques. 


\subsection{Taille de guêpe?}

Le champ lexical animalier est décidément propice aux jeux de mots ; Radio Days en offre une seconde illustration lors du jeu radiophonique cité plus haut :

V0, 66'15, MC: And what do you do, Bea?

Bea: I'm a book-keeper.

MC: Oh, for a minute, I thought you'd said beekeeper. l'd hate to get stung.

Le maître de cérémonie s'essaie à une plaisanterie aimable en direction de Bea, basée sur sa qualification professionnelle et la similitude phonétique de son prénom avec le nom commun bee, "abeille " en français, mais la paronymie entre book-keeper, comptable, et beekeeper, apicultrice, ne peut être transposée par le biais d'une traduction littérale en français. La difficulté est renforcée du fait du développement qui clôt sa répartie : l'd hate to get stung (je ne voudrais pas me faire piquer), accueilli par les rires embarrassés de Bea et du public diégétique. Cette réaction des personnages impose la production d'un effet similaire au doublage, d'où l'abandon du contenu sémantique :

VD, Animateur: Et que faites-vous dans la vie, Bea?

Bea: Je suis chef comptable.

Animateur: Oh, j'ai eu peur que vous ne disiez bea-romane, parce que je risquais de m'enflammer!

La traduction retenue joue sur la prononciation américaine du prénom de Bea, intégré dans un mot valise, " bea-romane ». Freud (1988, 62-63) qualifie ce type de construction par amalgame de " mot mixte, $[. .$.$] incompréhensible en lui-même, mais instantanément compris et identifié$ comme un mot plein de sens dès qu'il apparaît dans son contexte ". Ici, le néologisme entretient une relation paronymique avec le nom "pyromane ", qui permet de filer la métaphore jusqu'à la fin de la réplique en anticipant le verbe " enflammer ". Le jeu de mots, plus laborieux qu'en V0, ne dénature pas la caractérisation de l'animateur, habitué de ce type de plaisanteries, on l'a constaté précédemment. Cette stratégie répond à deux impératifs quelquefois contradictoires, traduction homomorphe et équivalence pragmatique, et reste conforme à l'horizon d'attente des spectateurs.

\section{Duplicité homophonique}

L'homophonie, autre phénomène, autorise de multiples jeux de mots, car elle marque la relation entre des lexèmes qui diffèrent quant à leur graphie mais partagent une prononciation commune - "le jeu de mots surexploite la langue par les divers sens auxquels prêtent les segments sonores, modifiés ou non "(Dupriez, 1984, 201). Cette identité phonétique est 
d'autant plus ambiguë en matière de doublage : les possibilités de discrimination y demeurent strictement auditives pour les références extra-diégétiques et ne peuvent s'appuyer sur la lecture comme en traduction littéraire.

\subsection{Un nickel pas très brillant}

Dans certains cas, le jeu de mots, déjà ardu en V0, le devient plus encore en langue d'arrivée. Dans Annie Hall, Alvy revoit en flash-back une scène de son enfance où un ami de la famille se présente avec insistance :

V0, 60', 148-150, Joey: Joey Nichols. (Laughing) See, Nichols. See, Nichols! (Joey shows young Alvy his cuff links and tie pin, which are made from nickels, as Alvy stands with hands on hips, unconcerned. Joey then slaps his hand to his forehead and puts a nickel on his forehead) Yuh see, nickels! You can always remember my name, just think of Joey Five Cents. (Laughing) That's me. Joey Five Cents!

La plaisanterie est construite sur l'homophonie Nichols/nickels. Nickel est le nom familier de la pièce américaine de cinq cents, et la réplique offre ainsi en V0 une sorte de traduction intralinguistique destinée au jeune interlocuteur de Joey. Elle signe la mauvaise qualité de la plaisanterie, puisque cette duplicité phonique devrait se comprendre d'elle-même.

DV, 39, Joey Nichols: (il lui pince la joue) Joey Nichols. (II lui montre son épingle à cravate.) Tu vois, Nichols. (II lui montre ses boutons de manchettes.) Tu vois, Nichols ! (II rit... et se colle une pièce(13) sur le front.) Tu vois, nickels ! Tu te rappelleras toujours de moi pense à Joey Cinq Cents. (II rit.) C'est moi. Joey Cinq Cents !

Valion choisit la traduction littérale en ajoutant une note de bas de page pour permettre au lecteur de décoder le jeu de mots : "(13) A Nickel: une pièce de monnaie en nickel qui valait cinq cents. "Dans cette traduction publiée dans une revue, la stratégie vise l'appréhension du texte par la lecture : elle met en avant le sémantisme verbal et les indications techniques, loin des priorités d'une traduction audiovisuelle. Dutter, lui, opte pour une formulation encore plus directe :

GD, 149-151, Joey montre son épingle de cravate et ses boutons de manchette, qui sont faits de pièces, à Alvy, totalement indifférent. Joey Nichols : Joey Nichols. Tu vois, Nichols. Tu vois, Nichels ! (sic) (II rit) Joey se plaque la main sur le front, un nickel y reste collé. Tu vois, nickels ! Tu te rappelleras toujours de moi, pense à Joey Nickels ou Cinq Cents. (II rit.) C'est moi. Joey Cinq Cents !

Ici, la traduction rétablit l'analogie Nickels = Cinq Cents en coordonnant les deux termes avec la conjonction " ou ». Cette construction syntaxique assure la transition d'un terme à l'autre(10)

(10) La coordination est une "relation entre deux éléments [...] qui ont le même statut dans l'énoncé ", au sein de laquelle la conjonction or a une valeur disjonctive, car elle installe le second comme une alternative au premier. Jacqueline Guillemin-Flescher, 1981, Syntaxe comparée du français et de l'anglais, Gap, Ophrys, p. 425, 441. 
en fonctionnant comme une quasi incrémentialisation (explicitation ou paraphrase insérée dans le texte même), sans perturber le rythme global de la tirade, ni le synchronisme kinésique. Cette stratégie diffère de celle choisie par Valion pour respecter la préconisation courante quant à l'homophonie : "Lorsqu'elle est exploitée volontairement, elle engendre une plurivalence qui doit être rendue en traduction " (Henry, 2003, 290). Le doubleur va être plus explicite encore sur cette duplicité référentielle nickel/Nichols puisque le public français entend en VD :

VD, Joey Nichols: Joey Nichols. Tu vois, Nichols. Tu vois, Nichols ! Eh, eh ! Ça, c'est un nickel ! C'est Cinq Cents. Et tu pourras jamais oublier mon nom, parce que tu diras : c'est Joey Cinq Cents ! C'est moi, Joey, la p'tite pièce de cinq cents !

Le présentatif "c'est ", martelé à quatre reprises, dirige le regard du spectateur vers la pièce exhibée à l'écran, de façon très insistante (la première occurrence est même précédée du pronom clitique " ça ") ; il s'accompagne de deux occurrences de " tu vois "à destination du personnage d'Alvy enfant : on a dans la VD tout à la fois monstration(11) et désignation, et le doublage exploite pleinement le phénomène d'audio-vision décrit par Chion $(2005,11)$ : “C'est la structuration même de la vision qu'elle engage, en la cadrant rigoureusement ". Les fonctions dramatique (caractérisation du personnage) et culturelle (respect du contexte civilisationnel) sont assurées en VD, mais celle-ci accentue "la valeur ajoutée du texte sur l'image " (ibid.) comparée à la V0. Cet effet de redondance révèle ainsi le doublage comme support possible de manipulation du spectateur, même si, dans cet exemple, il ne fait qu'intensifier un trait déjà relevé en VO.

Ces divers exemples permettent en tout cas de constater que le paradigme de l'intraduisibilité des jeux de mots est infirmé par la pratique, même dans un contexte aussi contraint que celui du doublage. Les différentes solutions mises en œuvre dans les versions françaises résultent souvent de choix entre plusieurs niveaux d'équivalence, mais elles réussissent en général à privilégier ce marqueur stylistique si prégnant chez Woody Allen, l'humour verbal, basé dans les extraits cités ci-dessus sur la "duplicité des jeux de mots " (Henry, 2003, 41). Celle-ci est redoublée lors du transfert linguistique et la figure de l'humoriste Allen est ainsi préservée en VD, car les jeux de mots font partie intégrante de son système d'écriture et donc de l'horizon d'attente de ses spectateurs francophones.

Certaines stratégies traductives vont encore plus loin et instaurent cette visée en VD alors qu'elle était absente de la V0, selon un processus de traduction créative illustré dans notre dernière partie.

(11) "La monstration, c'est-à-dire la mise en évidence, est une des forces du discours : dans un souci phatique (contact immédiat avec le public), pédagogique (clarté du propos) et rhétorique (efficacité des discours). [...] Si les démonstratifs signalent en effet des choses, ils exhibent surtout le locuteur en train de s'accomplir ", Damon Mayaffre, 2014, " "ça suffit comme ça !" La fausse opposition quantitatif/ qualitatif à l'épreuve du discours sarkozyste ", Corela, hs n 15, consulté le 27 avril 2015. http://corela.revues.org/3543 


\section{Duplicité créée}

En tant que technique de substitution, le doublage rend possible l'introduction dans le texte cible de répliques ironiques là où le dialogue originel n'en comprenait pas. Cette option peut parfois résulter d'un désir de compensation de l'adaptateur, sans que ce soit obligatoirement sa motivation première. Henry $(2007,192)$ considère du reste qu'à ce niveau " on atteint les limites extrêmes de la traduction des jeux de mots [...] la traduction libre ". Elle précise néanmoins que la démarche se justifie en fonction du " contexte cognitif local ou global " ou du " contexte verbal (présence de certains termes dans le texte ou encore de jeux de mots, ou, tout au moins, de figures d'écriture particulières) "; si la formulation diverge, ces conditions procèdent toutefois de l'intentio operis (Eco, 2004, 5), intention du texte au sens large qui implique l'appréhension de celui-ci à partir de ses mécanismes internes.

Les dialogues, et plus globalement les " textes filmiques " d'Allen, répondent aux critères d'Henry cités ci-dessus ; deux exemples relevés dans Deconstructing Harry, film caractérisé par sa grande causticité, sont présentés pour clôre notre étude de cas. Ce ne sont pas à proprement parler des jeux de mots, puisqu'ils portent sur des segments plus étendus qu'un seul lexème, mais ils jouent également sur le phénomène de duplicité, en exploitant le double sens d'expressions figées et procèdent donc de la même démarche, car " c'est quand l'esprit parvient à enfermer le langage dans ses propres contradictions que triomphe le jeu de mots, quelle qu'en soit la forme " (Dupriez, 1984, 269).

\subsection{Problèmes digestifs}

Lors d'une bar-mitsva, séquence de fiction extraite d'un roman de Harry, l'écrivain héros du film éponyme, Dolly Pincus découvre que Max, son époux, marié une première fois avant leur rencontre, avait assassiné puis dévoré son ex-famille. Son amie Elsie émet cette recommandation:

V0, 57'55, Elsie: Maybe the, the best course of action is to let sleeping dogs lie.

La réplique établit une analogie peu flatteuse entre Max et l'espèce canine. Cette métaphore lexicalisée, synonyme de not make someone angry, not make trouble (Dictionary of American Idioms) se traduit littéralement par " laisser tranquilles les chiens qui dorment ", et la locution française est très proche, "ne pas réveiller le chat qui dort ", mais ni la VF ni la VD ne retiennent cette option.

JC, 129, VD, Elsie : Peut-être la meilleure chose à faire, c'est de digérer ça, un peu en silence.

La métaphore digestive fait écho pour le spectateur au cannibalisme dévoilé quelques minutes plus tôt et s'avère cruellement ironique. La traduction de Cohen, basée sur le phénomène fort bien-nommé en anglais de double entendre, installe dans le dialogue une connotation absente 
de la VO à cet instant précis, mais s'insère facilement dans le contexte du récit et ne perturbe pas le spectateur confronté à une intrigue extrêmement grinçante depuis l'ouverture du film. Le doubleur a d'ailleurs retenu cette même stratégie.

\subsection{Plat de résistance}

Cohen n'a pas hésité à amplifier l'effet, avec un second exemple dans le même titre, quand Max réagit à la découverte de son crime par Dolly :

V0, 59'15, Max: So, what are you making a fuss? Some bury, some burn... I ate.

JC, 131, Max: Alors pourquoi tu en fais tout un plat ? II y en a qui enterrent, il y en a qui brûlent, moi, j'ai mangé !

VD, Max : Alors pourquoi tu en fais tout un plat ? Certains enterrent, d'autres brûlent, j'ai mangé !

En filant la métaphore alimentaire avec " en faire tout un plat ", synonyme de " en faire toute une affaire " d'après Le Robert, l'adaptatrice joue une nouvelle fois sur la polysémie d'une expression idiomatique pour traduire make a fuss, équivalent argotique de make trouble (Dictionary of American Idioms), une minute vingt après le précédent exemple ; suivie sur ce point par le doubleur, elle affiche une réelle cohérence, en exploitant le registre allusif. La banalité du cliché, destinée à relativiser la portée du crime dans la bouche de son auteur, accentue par contraste l'horreur de celui-ci. Le jeu de mots du cannibale présumé, bien que pure création de Cohen dans la VD, est porteur d'un humour noir très allenien, en accord avec le co-texte (la mention some burn, dans la bouche d'un Juif(12) assez âgé pour avoir connu la Shoah, est redoutable) et il vise le Spectateur Modèle construit par le texte filmique initial, dans le respect de l'intentio operis: de Baecque $(1998,7)$, dans son introduction au scénario publié, souligne " le vertige incessant et le comique kafkaïen " de ce film, qui permet à Allen de " donner par le malaise et par l'angoisse [...] une rageuse leçon de vitalité ", leçon parfaitement comprise et partagée ici par Cohen.

Comme le rappelle Bergson (2002, 86, souligné par l'auteur) : "On obtiendra un mot comique en insérant une idée absurde dans un moule de phrase consacré. [...] Et comme il y a des phrases toutes faites dans toutes les langues, [il] est généralement transposable, quoiqu'il soit rarement traduisible. " Les deux exemples ci-dessus jouent sur la polysémie de locutions figées, qui conduit l'adaptatrice à exploiter cette donnée linguistique en inscrivant dans la VD des jeux de mots conçus ex nihilo. Díaz Cintas $(2001,205)$ se demande si la portée connotative de l'ironie allenienne n'est pas vouée à disparaître à la traduction. Or on trouve ici l'illustration du phénomène inverse, grâce aux possibilités qu'offre le doublage, de par sa transparence et par-delà la " fidélité linguistique » invoquée par Cary $(1985,66)$ dans son interrogation sur ce mode de traduction audiovisuelle :

(12) Max, comme Elsie dans l'exemple 5.1., s'exprime avec un accent yiddish caricatural, et une minute après cette scène, le beau-frère de Harry résume ainsi les écrits de ce dernier : He creates offensive, Jewish stereotypes. 
Si l'on prétend fonder objectivement la notion de traduction sur des critères linguistiques et agréer ou rejeter les genres selon leur degré de fidélité linguistique à l'original, le doublage ne pourrait-il pas revendiquer au contraire une primauté dans la fidélité ?

\section{Conclusion}

Les traducteurs-adaptateurs mettent donc en œuvre les stratégies les plus variées : que ce soit pour les jeux de mots à base strictement phonétique ou pour les répliques plus subtiles, fondées sur l'allusion et l'ironie, leur pratique démontre que le transfert interlinguistique se révèle non seulement possible mais souvent très astucieux. On a également constaté que leur créativité peut les amener, au-delà de la traduction isomorphe, à l'application de solutions homomorphes ou hétéromorphes. Ils ne sauraient souffrir du travers déploré par Meschonnic $(2007,29)$ : "Quelle que soit la terminologie, le comportement est le même, l'attention à la langue de départ est une attention à la forme, l'attention à la langue d'arrivée, une attention au contenu. " Ici, le souci de la forme prédomine en VD, car il est essentiel pour l'efficacité des jeux de mots. Dutter, dans sa postface à la publication française de quatre scénarios d'Allen (1981, 446), rappelait l'ambiguité qui préside au travail de l'adaptateur :

Ce qui précède n'est pas tout à fait une traduction. C'est une transcription du texte original, avec les nécessaires transpositions, une translittération... une transe, en tout cas. On peut ainsi être assuré d'entendre, en français, le phrasé de Woody Allen, grand clarinettiste.

Je veux bien dire... sa musique.

II soulignait ainsi les limites de ce mode de traduction audiovisuelle, tout en validant son propre projet traductif : faire entendre Woody Allen. Dans cette démarche, le transfert des jeux de mots, souvent fondés sur des phénomènes sonores, est primordial. Dutter, puis Cohen, ne s'y sont pas trompés. Les adaptateurs confirment ainsi le postulat selon lequel "le doublage cinématographique est un travail de dialoguiste, une opération spécifiquement cinématographique, qui déborde la linguistique " (Mounin, 1976, 14). Dans les cas extrêmes, leurs VD vont jusqu'à offrir au public cinéphile francophone des occurrences supplémentaires de ces jeux sur la duplicité verbale. Grâce à l'attention très fine portée au transfert de ces usages ludiques de la langue récurrents dans l'œuvre d'Allen, dans le respect de l'intentio auctoris et de l'intentio operis, les traducteurs de doublage contribuent à démentir les a priori péjoratifs qui s'appliquent encore trop souvent au travail d'adaptation, et à réjouir les spectateurs francophones du cinéaste américain. 


\section{Bibliographie}

ALLEN Woody, 1977, Annie Hall, trad. D. Valion, L'Avant-Scène Cinéma, 198, p. 7-52.

ALLEN Woody, 1981, Opus 9-10-11-12, trad. et postface G. Dutter, Paris, Solar.

ALLEN Woody, 1990, Tout ce que vous avez toujours voulu savoir sur le sexe sans jamais oser le demander, trad. M. Lebrun, Paris, Éditions du Seuil.

ALLEN Woody, 1998, Deconstructing Harry, trad. J. Cohen, Paris, Cahiers du cinéma.

ALLEN Woody, BRICKMAN Marshall, 2000, Annie Hall, trad. G. Dutter, Paris, Cahiers du cinéma.

BAXTER John, 1999, Woody Allen, a Biography, London, HarperCollins.

BENAYOUN Robert, 1985, Woody Allen au-delà du langage, Paris, Herscher.

BERGSON Henri, 2002 [1940], Le rire, essai sur la signification du comique, Paris, PUF.

BERMAN Antoine, 1984, L'épreuve de l'étranger, Paris, Gallimard.

CARY Edmond, 1985, Comment faut-il traduire? ? Lille, Presses Universitaires de Lille.

CHION Michel, 2005 [1990], L'audio-vision, son et image au cinéma, Paris, Armand Colin.

CORNELOUP Pierre, 2011, "L'art du doublage : Emmanuel Fouquet, homme de paroles ", Vie Nouvelle, 164, p. 34-35.

DESMOND William, 2005, Paroles de traducteur, Louvain, Peeters.

DÍAZ CINTAS Jorge, 1998, "The Dubbing and Subtitling into Spanish of Woody Allen's Manhattan Murder Mystery ", Linguistica Antverpiensia, 32, p. 55-71.

DÍAZ CINTAS Jorge, 2001, "Striving for quality in subtitling: the role of a good dialogue list ", in GAMBIER Yves, GOTTLIEB Henrik, (eds.), (Multi) Media Translation: Concepts, Practices, and Research, Amsterdam, John Benjamins, p. 199-211.

DUPRIEZ Bernard, 1984, Gradus, les procédés littéraires, Paris, 10/18.

DURASTANTI Sylvie, 2002, Éloge de la trahison, notes du traducteur, Paris, Le Passage.

ECO Umberto, 2004, Mouse or Rat? Translation as Negotiation, London, Phoenix.

ECO Umberto, 2006, Dire presque la même chose, expériences de traduction, trad. Myriem Bouzaher, Paris, Grasset \& Fasquelle.

FREUD Sigmund, 1988, [1940], Le mot d'esprit et sa relation à l'inconscient, Paris, Gallimard. HENRY Jacqueline, 2003, La traduction des jeux de mots, Paris, Presses Sorbonne Nouvelle. 
MESCHONNIC Henri, 2007, Éthique et politique du traduire, Lagrasse, Verdier.

MOUNIN Georges, 1976, Les problèmes théoriques de la traduction, Paris, Gallimard.

RAYNAUD Jean, 1977, "Des mécanismes du jeu de mots ", RFEA, 4, p. 21-30.

SUHAMY Henri, 1994, Stylistique anglaise, Paris, PUF.

Frédérique Brisset, agrégée d'anglais et maître de conférences, enseigne la traduction et la traductologie à l'université Lille 3 Charles de Gaulle (EA CECILLE). Ses recherches portent sur le doublage de l'anglais américain vers le français et son impact sur la construction de l'image de l'auteur et sa réception par le public. Elle s'appuie sur l'approche traductologique contrastive et les théories de l'esthétique de la réception appliquées au cinéma. Elle a communiqué et publié en France et à l'étranger sur la cohérence en traduction dialogique, l'homogénéisation en traduction audiovisuelle, le doublage des emprunts, des noms propres et de l'humour, l'influence du marketing sur la traduction des titres de films, ainsi que sur les phénomènes de sérialité chez Woody Allen. Elle travaille actuellement sur le générique comme paratexte filmique, la traduction des anthroponymes politiques et celle de l'implicite lié à l'ironie. 